\title{
El consumo de drogas en estudiantes de México: tendencias y magnitud del problema
}

\author{
Jorge Ameth Villatoro Velázquez, ${ }^{1}$ Ma. Elena Medina-Mora Icaza, ${ }^{2}$ Raul Martín del Campo Sánchez, ${ }^{3}$ \\ Diana Anahí Fregoso Ito, 'Marycarmen Noemí Bustos Gamiño, 'Esbehidy Resendiz Escobar,' \\ Roxana Mujica Salazar, ${ }^{1}$ Michelle Bretón Cirett, ${ }^{1}$ Itzia Sayuri Soto Hernández, ${ }^{1}$ Vianey Cañas Martínez'
}

Artículo original

\section{ABSTRACT}

\section{Introduction}

Drug use and its social and health consequences are subject of interest. Students' surveys are epidemiological strategies to monitor this problem, and they are complemented with household and specific population surveys. All this can show in advance some phenomenon that later is visible in general population. Besides drug use trends and patterns, these studies show the influence of other factors that may increase the drugs use and their consequences.

\section{Objective}

To know the drugs, alcohol and tobacco use prevalences in elementary (5th and 6th grade), middle and high school students (7th-12th grades) of Mexico and its States.

\section{Material and methods}

This survey is a probabilistic elementary-high school students' survey with national and statal representativity. The study sample had 52 171 elementary students and 114364 middle and high school students. The information was obtained with a previously standardized and validated questionnaire.

\section{Results}

The lifetime tobacco use was $30.4 \%$ and the average onset age was 13.4 years old. $53.2 \%$ has drunk alcohol and $14.5 \%$ has binge drinking. Any drug use lifetime prevalence is $17.2 \%$. The preferred drugs are marihuana $(10.6 \%)$, followed by inhalants $(5.8 \%)$ and cocaine (3.3\%).

\section{Discussion and conclusion}

The increase in drugs use, specially of marihuana and alcohol, highlights the importance of creating new prevention strategies focused to decrease their use rate among students.

Key words: Surveys and questionnaires, prevention \& control, drug users, students, Mexico.

\section{RESUMEN}

\section{Introducción}

El consumo de drogas y sus consecuencias sociales y de salud son del interés de múltiples sectores. Las encuestas en estudiantes son estrategias epidemiológicas para monitorear el problema, las cuales se complementan con encuestas de hogares y en grupos especiales de la población, lo que permite ver anticipadamente fenómenos que posteriormente son evidentes en la población general. Además de las tendencias y del patrón de consumo de drogas, estos estudios muestran la influencia de otros factores que incrementan el uso y sus consecuencias.

\section{Objetivo}

Conocer las prevalencias de uso de drogas, alcohol y tabaco en estudiantes de primaria $\left(5^{\circ}\right.$ y $\left.6^{\circ}\right)$ y secundaria-bachillerato de México y sus Estados.

\section{Método}

Es una encuesta probabilística en población escolar $5^{\circ}$ y $6^{\circ}$ de primaria, secundaria y bachillerato con representatividad nacional y de cada Estado del país. La muestra fue de 52171 alumnos en primaria y 114364 en secundaria-bachillerato. La información se obtuvo mediante un cuestionario estandarizado y validado en encuestas anteriores.

\section{Resultados}

El consumo de tabaco alguna vez fue de $30.4 \%$ y la edad promedio de inicio de esta sustancia fue 13.4 años. El $53.2 \%$ ha bebido alcohol y $14.5 \%$ ha bebido en exceso. La prevalencia de consumo de drogas alguna vez fue de $17.2 \%$. Las drogas de preferencia son la mariguana $(10.6 \%)$, seguida de inhalables $(5.8 \%)$ y cocaína $(3.3 \%)$.

\section{Discusión y conclusión}

El crecimiento del consumo de sustancias, principalmente mariguana y alcohol, resalta la importancia de la creación de nuevas estrategias de prevención para disminuir los índices de consumo entre los estudiantes.

Palabras clave: Encuestas y cuestionarios, prevención y control, usuarios de drogas, estudiantes, México.

Dirección de Investigaciones Epidemiológicas y Psicosociales, Instituto Nacional de Psiquiatría Ramón de la Fuente Muñiz.

Dirección General del Instituto Nacional de Psiquiatría Ramón de la Fuente Muñiz.

3 Comisión Nacional contra las Adicciones.

Correspondencia: Lic. Jorge Villatoro Velázquez. Dirección de Investigaciones Epidemiológicas y Psicosociales, Instituto Nacional de Psiquiatría Ramón de la Fuente Muñiz, Calz. México-Xochimilco 101, San Lorenzo Huipulco, Tlalpan, 14370, México, DF. Tel: 554160 - 5201. E-mail: ameth@imp.edu.mx

Recibido: 22 de abril de 2016. Aceptado: 22 de junio de 2016. 


\section{INTRODUCCIÓN}

El consumo de drogas y sus consecuencias sociales y para la salud son un tema de interés para múltiples sectores de la sociedad; una de las inquietudes más comunes es conocer de qué magnitud es el problema. Los estudios epidemiológicos contestan esta pregunta y nos informan de la extensión del consumo, de las variaciones en diferentes grupos y regiones del país y de las tendencias en el tiempo.

Los estudios epidemiológicos se iniciaron en México en la década de 1970 y fueron las encuestas de escuelas las que abrieron el camino y, desde 1975, el ahora Instituto Nacional de Psiquiatría Ramón de la Fuente Muñiz y la Secretaría de Educación Pública han venido desarrollando mediciones transversales en población estudiantil de enseñanza media y media superior.

En 1976 y 1986 se hicieron las primeras encuestas nacionales con población urbana, en 1991 se hizo la primera encuesta nacional con representación estatal que también incluyó población rural.

Las encuestas realizadas en población estudiantil son una de las múltiples estrategias epidemiológicas que se tienen para monitorear el problema, se complementan con encuestas de hogares y en grupos especiales de la población, con estudios en cárceles, en población que acude a los centros de tratamiento, con estrategias de vigilancia activa, con estadísticas sociales, de procuración de justicia y de salud y con estudios con enfoque cualitativo. ${ }^{1,2}$ Cada estrategia tiene sus fortalezas especiales y se requiere de la conjunción de todas para dar cuenta cabal del problema.

Estas herramientas han permitido evaluar un fenómeno dinámico, con períodos de aumento y decremento, que varían geográficamente y en diferentes grupos de la población. Nos han mostrado que en México el uso de drogas ha aumentado, y junto con ello la proporción de personas que se ven afectadas por el problema.

Si bien, desde 1991 no se había hecho otro estudio nacional, en el transcurso se han realizado diversas encuestas estatales, en particular en la Ciudad de México, donde desde 1997 han sido trianuales.

Estos estudios en la población escolar han permitido ver anticipadamente diversos fenómenos que posteriormente se hacen evidentes en la población general. De manera particular, sin considerar al tabaco y al alcohol, ha mostrado que la mariguana fue la primera droga de uso hasta principios de la decáda de 1980, entonces los inhalables pasaron a ocupar el primer lugar. La preferencia cambia nuevamente hacia principios de los años 1990 cuando la mariguana recupera el primer lugar. ${ }^{3}$ Asimismo, dan cuenta de los primeros logros de la política antitabaco y de la persistencia del abuso de alcohol como principal problema de consumo.

A lo largo de los años, en la población estudiantil se han observado cambios en las preferencias de sustancias, como el consumo de mariguana que era primero, y posteriormen- te, durante casi dos décadas, los inhalables estuvieron en primer término. Hacia la década de 1990 el consumo de mariguana recuperó el primer lugar, pero también se registró un crecimiento importante en el consumo de cocaína, que coincide con el cambio en las rutas de tránsito de esta droga proveniente de la región andina hacia los Estados Unidos; la droga dejó de pasar principalmente por el Caribe y se usaron las rutas de México que ya operaban para el trasiego de heroína. ${ }^{4}$

Las encuestas en las escuelas han documentado también el crecimiento paulatino del crack y las metanfetaminas que, a pesar de ello, se mantienen en niveles bajos de consumo en esta población. Sin embargo, la problemática más importante en años recientes, y que a la fecha se mantiene en las mujeres, es el crecimiento que se dio entre 2006 y 2009 en el consumo de inhalables, de manera que pasó a ser la principal sustancia de consumo en las mujeres, no obstante, hacia 2012 esta prevalencia se mantuvo estable. ${ }^{5}$

El consumo de mariguana, por su parte, ha tenido un crecimiento acelerado, principalmente en los hombres. Sin duda, la discusión sobre la posible legalización de la mariguana ha permeado en la sociedad civil y ha impactado en la disminución de la percepción de riesgo y en el incremento de su consumo. Estos niveles de percepción de riesgo son similares a los que se tienen sobre el consumo de alcohol. ${ }^{5}$

Además de las tendencias del consumo y del patrón de consumo por tipo de droga, estos estudios nos muestran la influencia de la exposición a la oportunidad de su uso y de otros factores del contexto que incrementan la probabilidad de utilización (v.g. la percepción de riesgo asociado con el consumo y las actitudes e intenciones conductuales hacia el uso). Nos informan también sobre las consecuencias del consumo (por ejemplo, pobre rendimiento escolar) y de otros problemas (como malestar emocional, conducta alimentaria de riesgo, acoso escolar, experiencias de abuso, intento suicida, problemas de conducta), así como su asociación con el problema de las drogas.

El presente artículo contiene información sobre la prevalencia de por vida, en el último año, por grupos de edad y sexo, población rural y urbana. Desde este contexto, se pretende contribuir al conocimiento de lo que ocurre en esta población y que sirva como herramienta de análisis para ampliar las acciones preventivas basadas en evidencia, no sólo en la población adolescente, sino en los niños más pequeños, con la finalidad de generar acciones reales de prevención y promoción de la salud.

\section{MÉTODO}

Este estudio mantuvo la metodología empleada y los aspectos básicos de las mediciones anteriores que se han realizado en estudiantes de nivel básico y medio superior en el país. Asimismo, es comparable con los indicadores epidemiológi- 
cos empleados en los estudios de América Latina (CICAD, OID), Monitoring the Future y ESPAD.

\section{Población y muestra}

El diseño de muestra comprendió tres estratos para cada uno de los Estados del país, así como para las nueve Ciudades Específicas del estudio, lo cual corresponde a los niveles educativos de primaria ( $5^{\circ}$ y $6^{\circ}$ grado), secundaria y bachillerato.

El marco muestral se obtuvo del registro oficial 20132014 de la Secretaría de Educación Pública de las escuelas de educación básica ( $5^{\circ}$ y $6^{\circ}$ de primaria), secundaria y media superior, los cuales se encontraban en una base de datos computarizada.

El diseño de muestra fue estratificado y por conglomerados, la variable de estratificación fue el tipo de escuela: primaria, secundaria y bachillerato, así como la Entidad Federativa y las nueve ciudades de interés (Acapulco, Apatzingán, Cancún-Playa del Carmen, Ciudad del Carmen, Ciudad Juárez, Cuautla, Ecatepec, Guadalajara y Tijuana). La unidad de selección fue el grupo escolar al interior de las escuelas. Se planteó por conglomerados (grupos) para optimizar los tiempos de los encuestadores y disminuir los costos del trabajo de campo. La muestra obtenida de grupos y alumnos se ponderó considerando el grupo, el nivel educativo y la Entidad Federativa o Ciudad, con objeto de realizar la estimación y el procesamiento de datos.

La estimación del tamaño se efectuó considerando una tasa de no-respuesta del $20 \%$ que es la encontrada en estudios previos y que incluyó el ausentismo por diversas causas. El nivel de confianza de la muestra es del 95\%, con un error absoluto promedio del .004 , con un efecto de diseño con valor 2 .

Con base en estos parámetros, se contempló tener una muestra de 1560 estudiantes en cada estrato (4680 en total por Estado) y 1560 estudiantes por estrato para cada una de las ciudades de interés (4680 en total por Ciudad). Las escuelas se seleccionaron aleatoriamente al interior de cada una de las 32 entidades y de las nueve ciudades de cada nivel educativo a través de un muestreo sistemático al interior de cada estrato. Esto da una estimación de casi 6358 grupos escolares o 191880 estudiantes encuestados (figura 1).

De manera simultánea a la obtención de la muestra principal, se obtuvo una muestra réplica con el mismo procedimiento, por lo que cada grupo en la muestra principal tuvo su equivalente en la muestra réplica. Los grupos de la muestra réplica se aplicaron únicamente en aquellos casos en que, por cuestiones de seguridad de la integridad física de los encuestadores o de los propios estudiantes, no fue posible realizar la aplicación. Si bien se aplicaron 61 grupos de la muestra réplica debido a situaciones de inseguridad en varios municipios del país, el efecto de no incluir estos grupos en la muestra final es mínimo sobre la tasa de res- puesta del estudio, por lo que se trabajó únicamente con los grupos de la muestra principal y se evitaron efectos desconocidos por incluir los 61 grupos de la réplica. Una mayor descripción del diseño de muestra puede verse en el reporte principal del estudio. ${ }^{6}$

\section{Instrumento}

La información se obtuvo mediante un cuestionario estandarizado, empleado en anteriores encuestas, que ha sido previamente validado. ${ }^{7-9}$

Debido a las características de la población escolar, se aplicó un cuestionario para primaria y otro para secundaria-bachillerato con algunas diferencias en su contenido.

El cuestionario de primaria se conformó por 12 páginas con las siguientes secciones: datos sociodemográficos, consumo de alcohol, tabaco, mariguana, inhalables y otras drogas; en el ámbito social se preguntó sobre tolerancia social al consumo de sustancias, la disponibilidad y la percepción del riesgo del consumo de drogas, de alcohol y de tabaco; su opinión acerca de las pláticas para prevenir el consumo de drogas que reciben en la institución escolar; así como su percepción de inseguridad (delincuencia y violencia) en la zona donde viven. En el ámbito interpersonal se preguntó sobre el consumo de drogas, los problemas por consumo de alcohol en la familia, la información acerca del uso de drogas que el alumno recibe en su hogar y las reglas en cuanto al uso de las mismas. En cuanto al grupo de pares, se inclu-

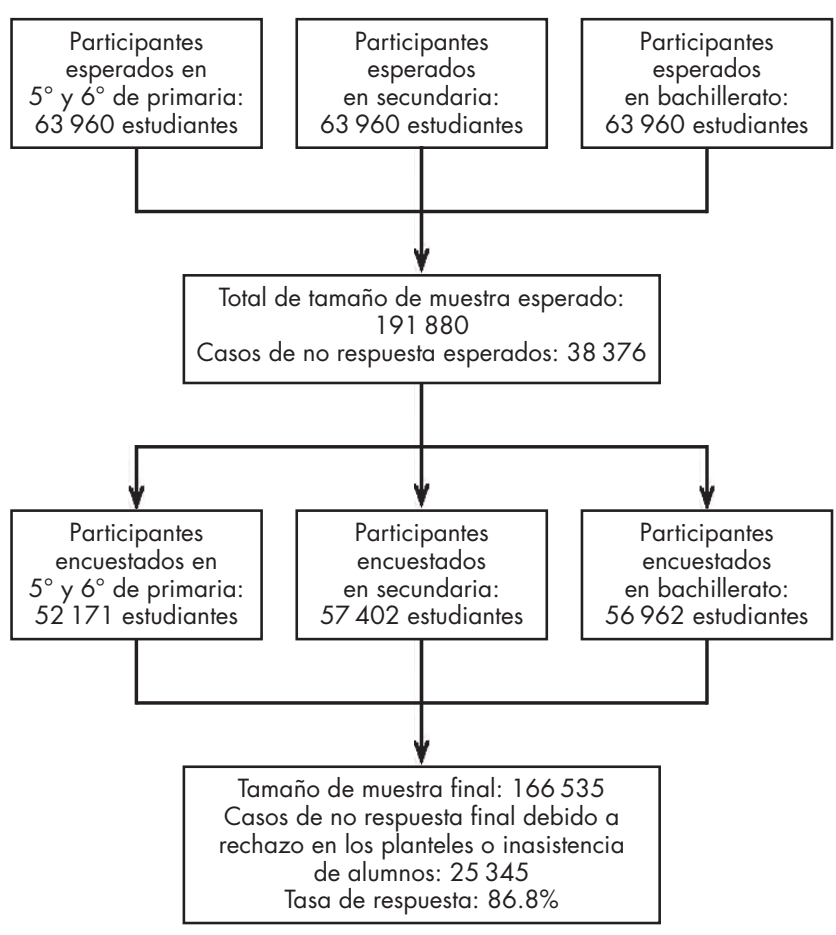

Figura 1. Diagrama del estudio. 
yeron preguntas sobre el consumo de drogas y alcohol en sus amigos, en diferentes contextos y sus características, así como la presión que ejercen sus amigos para que consuma sustancias. Finalmente, en el ámbito personal se preguntó acerca del desempeño del alumno, expectativas de vida, búsqueda de sensaciones, su nivel de autoestima, habilidades sociales con las que cuenta, razones para asistir a la escuela y a quién recurre cuando tiene algún problema.

En tanto, el cuestionario de secundaria y bachillerato se aplicó en cuatro formas con la finalidad de sondear una mayor variedad de factores relacionados. Una misma forma fue aplicada al grupo completo, pero la forma que se aplicó al grupo fue previamente aleatorizada sistemáticamente.

La sección principal que se aplicó a todos los sujetos contuvo preguntas sobre datos sociodemográficos, también se hicieron preguntas sobre el consumo de drogas, alcohol y tabaco, el gasto para comprar drogas y el consumo de drogas en su entorno. También se evaluaron las necesidades de apoyo o intervención por medio de la escala ASSIST, en la que se preguntó únicamente sobre la droga de mayor consumo. En el caso del alcohol, se incluyeron la versión corta y larga de la escala del AUDIT. En el ámbito social, se incluyeron preguntas sobre la tolerancia social, la disponibilidad y la percepción del riesgo del consumo de drogas, de alcohol y de tabaco, así como la percepción de inseguridad en la zona donde vive. En el ámbito interpersonal, se preguntó acerca de la composición familiar del sujeto y los hábitos de crianza; además, sobre el consumo de drogas y problemas por consumo de alcohol en la familia. En cuanto al grupo de pares, se incluyeron preguntas sobre el consumo de drogas y alcohol de los amigos del entrevistado en diferentes contextos. En el ámbito personal, se indagó su nivel de autoestima, de ajuste social, sus expectativas de vida, intento suicida y abuso sexual, las situaciones a las que se enfrenta el estudiante y violencia entre compañeros en la institución educativa, así como sobre la conducta antisocial del adolescente.

Los contenidos de las formas pueden consultarse en el reporte principal del estudio. ${ }^{6}$

\section{Consideraciones éticas}

El proyecto fue autorizado por el Comité de Ética para la investigación. Un aspecto relevante es que en las instrucciones iniciales del instrumento se indicó claramente que el sujeto podía aceptar o no la aplicación del cuestionario, o bien que podía dejar de contestarlo cuando lo considerara conveniente. Así mismo, en todo momento, dado el carácter grupal de la aplicación, se aseguró la confidencialidad y el anonimato del alumno.

\section{Análisis de datos}

Con objeto de obtener los intervalos de confianza de las prevalencias y encontrar en qué Estados y niveles educativos son menores o mayores a las prevalencias nacionales, se usó el programa STATA versión 13.

Los datos presentados en este artículo, debido a la extensión de la información del estudio, corresponden sólo a la información nacional y estatal. Las ciudades pueden consultarse en el informe general de la encuesta. ${ }^{6}$
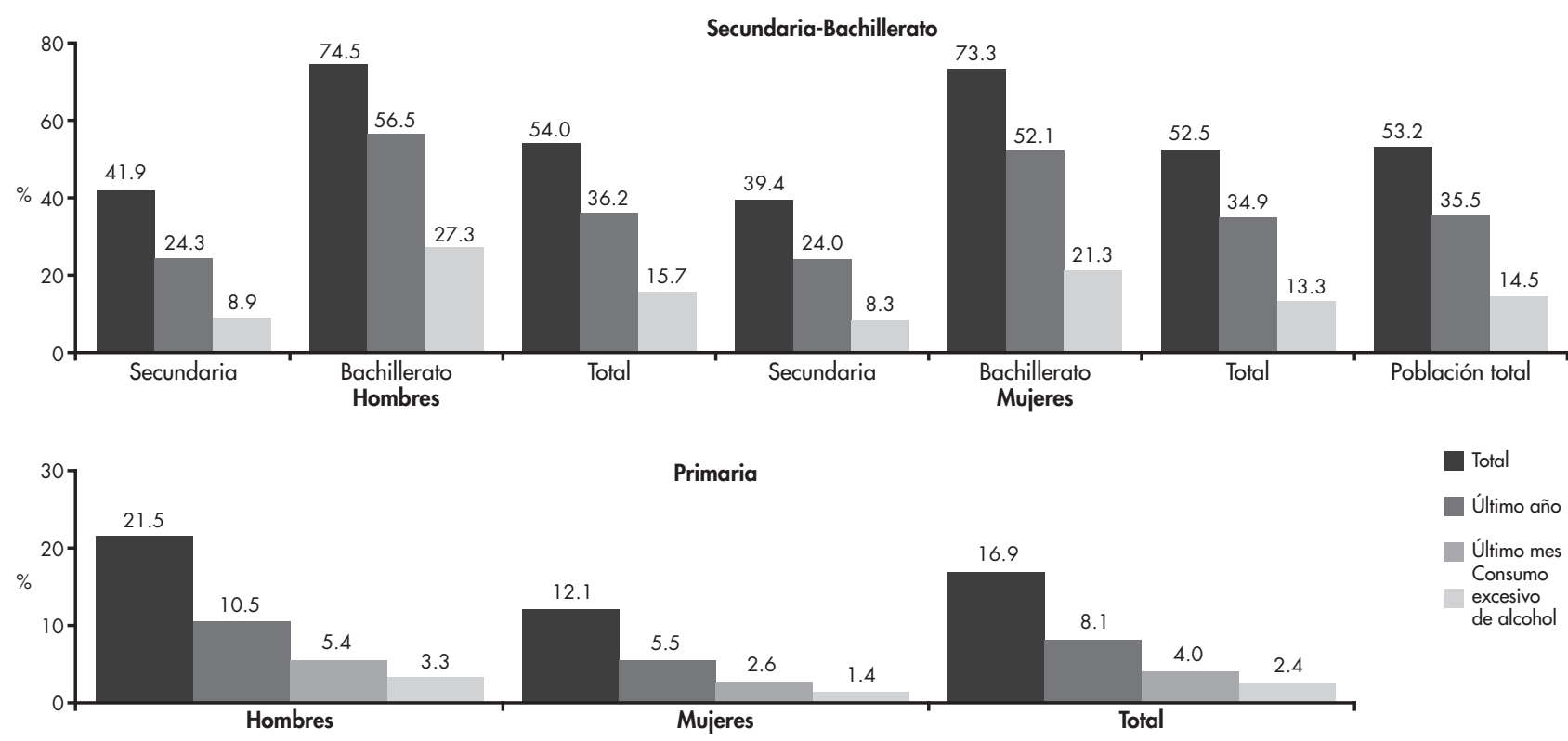

Fuente: Encuesta Nacional de Consumo de Drogas en Estudiantes, 2014

Figura 2. Prevalencia del consumo de alcohol en estudiantes. 


\section{RESULTADOS}

\section{Prevalencias del consumo de alcohol}

A nivel nacional, más de la mitad de los estudiantes de secundaria y bachillerato han bebido alcohol alguna vez en su vida (53.2\%), en los hombres el consumo fue de $54 \%$ y en las mujeres de $52.5 \%$. En el último año, el 35.5\% de los jóvenes bebió alcohol (36.2\% hombres y $34.9 \%$ mujeres) (figura 2); por nivel educativo, la prevalencia pasa de secundaria a bachillerato de $24.2 \%$ a $54.3 \%$.

El consumo excesivo de alcohol (cinco copas o más en una sola ocasión en el último mes) se presenta en el 14.5\% de los estudiantes (15.7\% hombres, $13.3 \%$ mujeres) (figura 2); por nivel educativo, $8.6 \%$ de los estudiantes de secundaria lo indica, mientras que para bachillerato el porcentaje se incrementa a $24.2 \%$. Los Estados que muestran prevalencias de consumo excesivo mayores a la reportada en el país son: Ciudad de México (19.5\%), Durango (17.7\%), Jalisco (17.4\%) y Michoacán (18.7\%) (cuadro 1).

$\mathrm{Al}$ analizar el consumo problemático, los resultados indican que el $14.4 \%$ de los estudiantes lo presenta $(16.5 \%$ hombres y $12.4 \%$ mujeres). Por nivel educativo, en secundaria $9.4 \%$ y en bachillerato $22.7 \%$.

La edad promedio de inicio de consumo de alcohol es de 12.6 años; por sexo, los hombres reportaron iniciar su consumo a los 12.4 años y las mujeres a los 12.8 años.

Por tipo de comunidad, se observa que el consumo "alguna vez" en comunidades rurales $(39.6 \%)$ es significativamente menor al reportado en comunidades urbanas (55.7\%); del mismo modo, el consumo de alcohol en el último año (37.2\% urbanas, $26.1 \%$ rurales), en el último mes (27.8\% urba-

Cuadro 1. Indicadores de consumo de sustancias psicoactivas por entidad federativa en estudiantes de secundaria y bachillerato

\begin{tabular}{|c|c|c|c|c|c|c|}
\hline & $\begin{array}{c}\text { Consumo } \\
\text { excesivo alcohol }\end{array}$ & $\begin{array}{c}\text { Tabaco } \\
\text { último mes }\end{array}$ & $\begin{array}{l}\text { Cualquier droga } \\
\text { alguna vez }\end{array}$ & $\begin{array}{l}\text { Mariguana } \\
\text { alguna vez }\end{array}$ & $\begin{array}{l}\text { Inhalables } \\
\text { alguna vez }\end{array}$ & $\begin{array}{l}\text { Cocaína } \\
\text { alguna vez }\end{array}$ \\
\hline Estado & $\%$ & $\%$ & $\%$ & $\%$ & $\%$ & $\%$ \\
\hline Aguascalientes & 14.5 & 10.9 & 16.1 & 9.6 & 5.4 & 2.5 \\
\hline Baja California & $11.1^{-}$ & $6.3^{-}$ & $14.2^{-}$ & 9.4 & $3.7^{-}$ & $2.5^{-}$ \\
\hline Baja California Sur & 13.5 & $8.1^{-}$ & 14.9 & 10.0 & $4.0^{-}$ & $1.8^{-}$ \\
\hline Campeche & $11.0^{-}$ & 9.1 & 16.2 & 8.7 & 4.8 & 3.4 \\
\hline Chiapas & $9.4^{-}$ & $7.5^{-}$ & $11.4^{-}$ & $5.5^{-}$ & $4.1^{-}$ & 3.0 \\
\hline Chihuahua & 16.9 & 11.1 & $19.8^{*}$ & 11.9 & 5.1 & 4.4 \\
\hline Coahuila & 16.5 & $13.4^{*}$ & 16.3 & 9.9 & 4.6 & $2.2-$ \\
\hline Colima & 17.0 & $8.6^{-}$ & 16.4 & 10.9 & $3.9-$ & 3.0 \\
\hline D.F. (Ciudad de México) & $19.5^{*}$ & $17.2^{*}$ & $25.0^{*}$ & $18.2^{*}$ & $8.0^{*}$ & $5.2^{*}$ \\
\hline Durango & $17.7^{*}$ & 11.4 & 15.3 & 8.8 & 5.2 & 2.8 \\
\hline Estado de México & 16.7 & $14.5^{*}$ & $21.1^{*}$ & $13.8^{*}$ & $8.8^{*}$ & 4.2 \\
\hline Guanajuato & 16.0 & 11.1 & 18.8 & 11.6 & 6.9 & 3.6 \\
\hline Guerrero & $11.3^{-}$ & 10.6 & 14.9 & $8.3^{-}$ & 5.2 & 3.6 \\
\hline Hidalgo & 16.0 & 11.6 & 16.0 & 9.4 & 6.3 & 3.2 \\
\hline Jalisco & $17.4^{*}$ & 10.7 & $20.1^{*}$ & 12.4 & 6.3 & 2.9 \\
\hline Michoacán & $18.7^{*}$ & 12.4 & 17.9 & 10.0 & 5.8 & 3.0 \\
\hline Morelos & 14.9 & $13.6^{*}$ & 19.2 & 12.3 & 6.2 & 3.1 \\
\hline Nayarit & 13.7 & $7.9-$ & 15.8 & 9.8 & $4.0^{-}$ & 2.8 \\
\hline Nuevo León & 13.1 & 10.9 & $14.5^{-}$ & $8.6^{-}$ & 5.0 & $2.4^{-}$ \\
\hline Oaxaca & $9.6^{-}$ & 7.7- & 15.2 & $8.5^{-}$ & 6.0 & 3.0 \\
\hline Puebla & 14.3 & 9.8 & 15.3 & $8.5^{-}$ & 5.2 & 3.1 \\
\hline Querétaro & 15.1 & 12.7 & 18.6 & 12.5 & 7.3 & 3.1 \\
\hline Quintana Roo & $11.8^{-}$ & 10.8 & $22.0^{*}$ & $14.6 *$ & 5.5 & $4.7^{*}$ \\
\hline San Luis Potosí & 13.5 & 9.1 & 16.0 & 10.1 & 5.5 & $2.5^{-}$ \\
\hline Sinaloa & $11.0^{-}$ & $4.6^{-}$ & $9.2^{-}$ & $4.9^{-}$ & $1.9-$ & $1.6^{-}$ \\
\hline Sonora & $11.8^{-}$ & $8.1^{-}$ & $13.0^{-}$ & 8.9 & $2.7^{-}$ & $2.2^{-}$ \\
\hline Tabasco & $11.1^{-}$ & $6.7^{-}$ & $10.6^{-}$ & $5.8^{-}$ & $2.6^{-}$ & 2.7 \\
\hline Tamaulipas & $10.5^{-}$ & $8.6^{-}$ & $13.7^{-}$ & 8.8 & $4.0^{-}$ & 4.1 \\
\hline Tlaxcala & 16.5 & 12.4 & $11.9-$ & $7.0^{-}$ & 4.8 & 2.7 \\
\hline Veracruz & $10.5^{-}$ & $8.4^{-}$ & 15.3 & $7.5^{-}$ & 6.1 & 2.7 \\
\hline Yucatán & $10.2^{-}$ & 11.5 & 15.6 & 8.9 & 4.9 & 2.7 \\
\hline Zacatecas & 15.1 & 10.8 & $13.4^{-}$ & $7.1^{-}$ & $3.4^{-}$ & $2.2^{-}$ \\
\hline Nacional & 14.5 & 11.1 & 17.2 & 10.6 & 5.8 & 3.3 \\
\hline
\end{tabular}

* Porcentaje significativamente mayor al obtenido a nivel nacional.

- Porcentaje significativamente menor al obtenido a nivel nacional. 
Villatoro Velázquez et al.

Cuadro 2. Necesidades de atención por consumo de alcohol, drogas o tabaco

\begin{tabular}{|c|c|c|c|c|c|c|}
\hline & \multicolumn{2}{|c|}{ Total } & \multicolumn{2}{|c|}{ Hombres } & \multicolumn{2}{|c|}{ Mujeres } \\
\hline & $N$ & $\%$ & $N$ & $\%$ & $N$ & $\%$ \\
\hline \multicolumn{7}{|c|}{ Necesidades de atención por consumo de alcohol } \\
\hline Riesgo alto & 167430 & 1.6 & 102833 & 2.0 & 64597 & 1.2 \\
\hline Riesgo medio & 206311 & 2.0 & 124504 & 2.4 & 81807 & 1.6 \\
\hline Riesgo bajo & 1135362 & 10.9 & 632693 & 12.2 & 502669 & 9.6 \\
\hline Sin riesgo & 8936602 & 85.6 & 4339972 & 83.5 & 4596630 & 87.6 \\
\hline \multicolumn{7}{|c|}{ Necesidades de atención por consumo de drogas } \\
\hline Requiere apoyo & 131258 & 1.3 & 79878 & 1.5 & 51380 & 1.0 \\
\hline No requiere apoyo & 9741851 & 93.2 & 4804243 & 92.3 & 4937608 & 94.1 \\
\hline $\begin{array}{l}\text { Consumo de tabaco } \\
\text { en los primeros } 30 \text { minutos* }\end{array}$ & 70563 & 9.1 & 44896 & 9.5 & 25667 & 8.5 \\
\hline
\end{tabular}

nas, $18 \%$ rurales) y consumo excesivo de alcohol (15.4\% urbanas, $9.7 \%$ rurales), presentan porcentajes menores de consumo en comunidades rurales en comparación con las urbanas.

$\mathrm{Al}$ indagar las necesidades de atención por consumo de alcohol, el $1.6 \%$ de los estudiantes (2\% hombres, $1.2 \%$ mujeres) presenta un riesgo alto por su consumo (cuadro 2).

El crecimiento de la problemática de 1991 al 2014 pasó de $29 \%$ a $35.5 \%$ para el consumo en el último año; mientras que para el consumo excesivo (cinco copas o más en una sola ocasión en el último mes) pasó de 9.5\% a 14.5\% (figura 3).

En los estudiantes de $5^{\circ}$ y $6^{\circ}$ de primaria, la prevalencia "alguna vez en la vida" es de 16.9\%, 21.5\% en los hombres y $12.1 \%$ en las mujeres (figura 2). El consumo de alcohol en el último año es de $8.1 \%$ (10.5\% hombres y $5.5 \%$ mujeres) y los Estados de Querétaro (12.5\%) y Tlaxcala (15.8\%) muestran porcentajes por arriba del nacional para este indicador.

En relación al consumo excesivo, el 2.4\% mencionó que en el último mes había consumido cinco o más copas en una sola ocasión (3.3\% hombres, $1.4 \%$ mujeres) (figura 2). Los Estados que tienen prevalencias mayores a las reportadas en el país son Querétaro (4.8\%) y Tlaxcala (4.2\%) (cuadro 3).

Al indagar la intención de consumir alcohol, el 12.5\% de quienes no han consumido indican que probablemente lo consumirán y $22.6 \%$ de quienes ya consumieron alcohol lo volverían a hacer.
Finalmente, por tipo de comunidad, la prevalencia total de consumo de alcohol es similar en los estudiantes de comunidades urbanas (17.1\%), y en los estudiantes de comunidades rurales $(16.1 \%)$, situación que también se observa en el consumo en el último año (8\% urbanas, $8.2 \%$ rurales), en el último mes (3.9\% urbanas, $4.4 \%$ rurales) y el consumo excesivo (3.1\% urbanas, $3.6 \%$ rurales).

\section{Prevalencias del consumo de tabaco}

El 30.4\% de los estudiantes ha fumado alguna vez en su vida, $34 \%$ hombres y $26.8 \%$ mujeres (figura 4 ); los porcentaje de consumo se incrementan significativamente en la transición de secundaria $(20.8 \%)$ a bachillerato $(46.2 \%)$. Los Estados con prevalencias superiores al nacional son Ciudad de México (39.9\%) y Estado de México (36.6\%).

En cuanto a la edad de inicio en el consumo de tabaco, los estudiantes reportaron 12.8 años; por sexo los hombres inician a los 12.6 años y las mujeres a los 13.1 años.

El porcentaje de fumadores actuales fue de $11.1 \%$ (13.3\% hombres y $8.9 \%$ mujeres) y el de fumadores diarios fue de $7.4 \%$ (9\% hombres y $5.8 \%$ mujeres) (figura 4 ). De estos fumadores diarios, el promedio de cigarros consumidos por día es alrededor de tres, tanto en hombres como en mujeres. Además, el 9.1\% (9.5\% hombres, 8.5\% mujeres) fuma su pri-

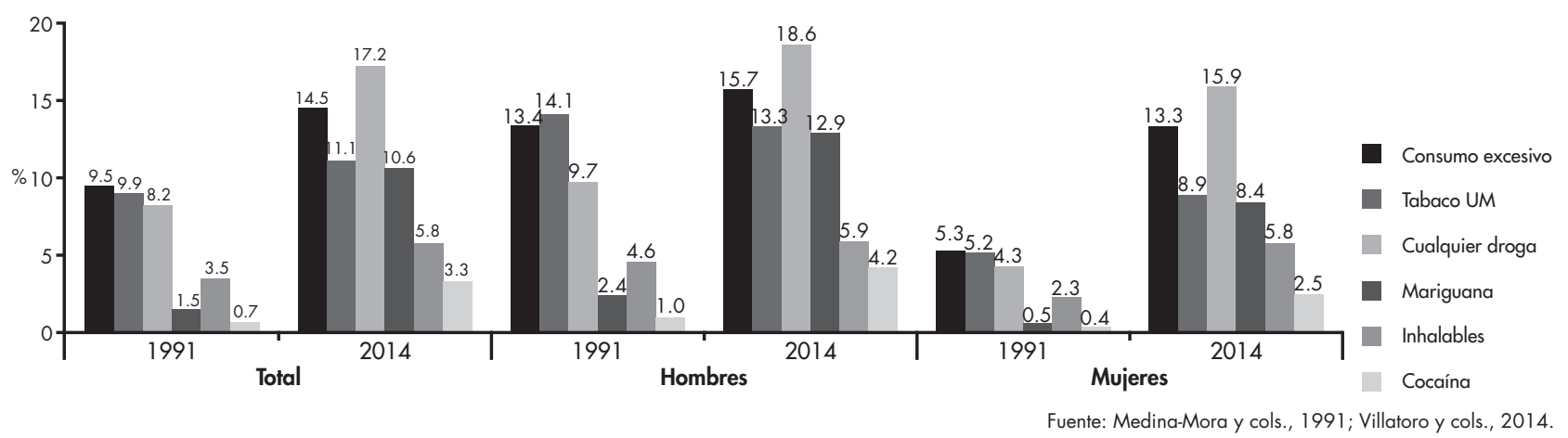

Figura 3. Tendencias en 23 años de consumo de drogas en estudiantes. 
Cuadro 3. Indicadores principales de consumo de sustancias psicoactivas por entidad federativa en estudiantes de primaria

\begin{tabular}{|c|c|c|c|c|c|}
\hline & $\begin{array}{c}\text { Consumo } \\
\text { excesivo alcohol }\end{array}$ & $\begin{array}{c}\text { Tabaco } \\
\text { último mes }\end{array}$ & $\begin{array}{l}\text { Cualquier droga } \\
\text { alguna vez }\end{array}$ & $\begin{array}{l}\text { Mariguana } \\
\text { alguna vez }\end{array}$ & $\begin{array}{l}\text { Inhalables } \\
\text { alguna vez }\end{array}$ \\
\hline Estado & $\%$ & $\%$ & $\%$ & $\%$ & $\%$ \\
\hline Aguascalientes & 3.3 & 3.5 & 3.3 & 2.5 & 1.6 \\
\hline Baja California & 1.5 & 2.7 & 4.1 & 2.8 & 2.5 \\
\hline Baja California Sur & 1.7 & 1.8 & 2.3 & 1.7 & 1.3 \\
\hline Campeche & 2.1 & 3.4 & $5.0^{*}$ & $3.5^{*}$ & 2.8 \\
\hline Chiapas & 1.4 & 2.4 & 3.5 & 2.3 & 2.0 \\
\hline Chihuahua & 2.2 & 2.0 & 3.7 & 2.6 & 1.8 \\
\hline Coahuila & 2.7 & 3.9 & 2.9 & 2.1 & 1.6 \\
\hline Colima & 2.6 & 2.6 & 4.4 & 3.3 & 2.3 \\
\hline D.F. (Ciudad de México) & 1.7 & $1.4-$ & 2.5 & 1.8 & $1.0-$ \\
\hline Durango & 2.8 & 2.6 & 3.8 & 3.1 & 2.1 \\
\hline Estado de México & 2.8 & 1.8 & 2.4 & 1.5 & 1.7 \\
\hline Guanajuato & 1.8 & 1.6 & $1.5-$ & $1.0-$ & $0.6-$ \\
\hline Guerrero & 3.3 & 4.0 & 5.0 & 4.0 & 2.9 \\
\hline Hidalgo & 3.0 & 2.0 & 3.4 & 1.8 & 2.4 \\
\hline Jalisco & 1.8 & 2.7 & 4.1 & 3.3 & 1.9 \\
\hline Michoacán & 3.5 & 3.4 & $5.3^{*}$ & $4.1^{*}$ & 2.7 \\
\hline Morelos & 2.0 & 3.1 & 3.7 & 2.6 & 2.0 \\
\hline Nayarit & 2.6 & 1.9 & 2.7 & 1.7 & 1.8 \\
\hline Nuevo León & 1.5 & 2.9 & 2.9 & 2.3 & 1.9 \\
\hline Oaxaca & 3.2 & 3.3 & 3.5 & 2.5 & 1.8 \\
\hline Puebla & 2.7 & 2.3 & 2.2 & 1.7 & $1.0-$ \\
\hline Querétaro & $4.8^{*}$ & $4.9 *$ & 4.3 & 3.0 & 2.7 \\
\hline Quintana Roo & 2.7 & $4.9^{*}$ & $5.9^{*}$ & $4.5^{*}$ & $3.4^{*}$ \\
\hline San Luis Potosí & 2.8 & 3.2 & $5.1^{*}$ & 3.5 & 2.5 \\
\hline Sinaloa & 1.6 & 2.2 & 2.9 & 2.3 & 1.6 \\
\hline Sonora & $0.8-$ & 1.9 & 3.4 & 1.8 & 2.9 \\
\hline Tabasco & 2.2 & 3.1 & 4.2 & 3.2 & 2.9 \\
\hline Tamaulipas & 1.6 & $1.3-$ & 2.9 & 2.2 & 1.7 \\
\hline Tlaxcala & $4.2^{*}$ & 3.7 & 2.8 & 2.1 & 1.7 \\
\hline Veracruz & 2.5 & 2.3 & 3.0 & 1.7 & 1.8 \\
\hline Yucatán & 2.8 & 3.6 & 4.3 & 3.1 & 2.2 \\
\hline Zacatecas & 1.9 & 3.2 & 2.9 & 2.0 & 1.3 \\
\hline Nacional & 2.4 & 2.5 & 3.3 & 2.3 & 1.8 \\
\hline
\end{tabular}

* Porcentaje significativamente mayor al obtenido a nivel nacional.

- Porcentaje significativamente menor al obtenido a nivel nacional.

mer cigarro en los primeros 30 minutos después de despertar por la mañana.

Los Estados con fumadores actuales que tienen porcentajes superiores al nacional son Distrito Federal (17.2\%), Estado de México (14.5\%), Morelos (13.6\%) y Coahuila (13.4\%) (cuadro 1); mientras que los Estados que presentan un consumo diario superior al nacional son Distrito Federal (11.1\%), Estado de México y Morelos (9.6\% cada uno).

Por otro lado, se observa una prevalencia total de consumo de tabaco significativamente mayor en los estudiantes de las comunidades urbanas (31.9\%) en comparación con los de las rurales $(22.1 \%)$, esto también se muestra en los diferentes indicadores de consumo, en el último año (81.5\% urbanas, $10.7 \%$ rurales), en el último mes (11.8\% urbanas, $7 \%$ rurales), consumo de 100 cigarrillos o más (4.5\% urbanas, $2.4 \%$ rurales) y consumo diario de tabaco ( $7.8 \%$ urbanas, $5.1 \%$ rurales).
Sobre la disponibilidad de los cigarrillos, en secundaria $45.3 \%$ de los fumadores indica que los compra en una tienda, $16.8 \%$ los consigue afuera de la escuela, 3\% los compra en la escuela, $3.3 \%$ los consigue con el permiso de sus padres y $43.5 \%$ sin su permiso. Para los estudiantes de bachillerato, $60.7 \%$ los compra en una tienda, $30.7 \%$ los consigue afuera de la escuela, $4.7 \%$ los compra en la escuela, $5 \%$ los consigue con el permiso de sus padres y $46.9 \%$ sin su permiso.

Las tendencias del consumo de tabaco entre 1991 y 2014 indican que el consumo se ha mantenido estable $(9.9 \%$ a $11.1 \%$ en el consumo en el último mes), aunque hay variaciones por sexo; mientras que en los hombres estuvo estable $(14.1 \%$ a $13.3 \%)$, en las mujeres se incrementó (5.2\% a 8.9\%) (figura 3).

La prevalencia del consumo de tabaco "alguna vez en la vida" en primaria es de $6.5 \%, 8.9 \%$ hombres y $4 \%$ mujeres (figura 4). El 2.5\% de los estudiantes refiere ser fumador ac- 

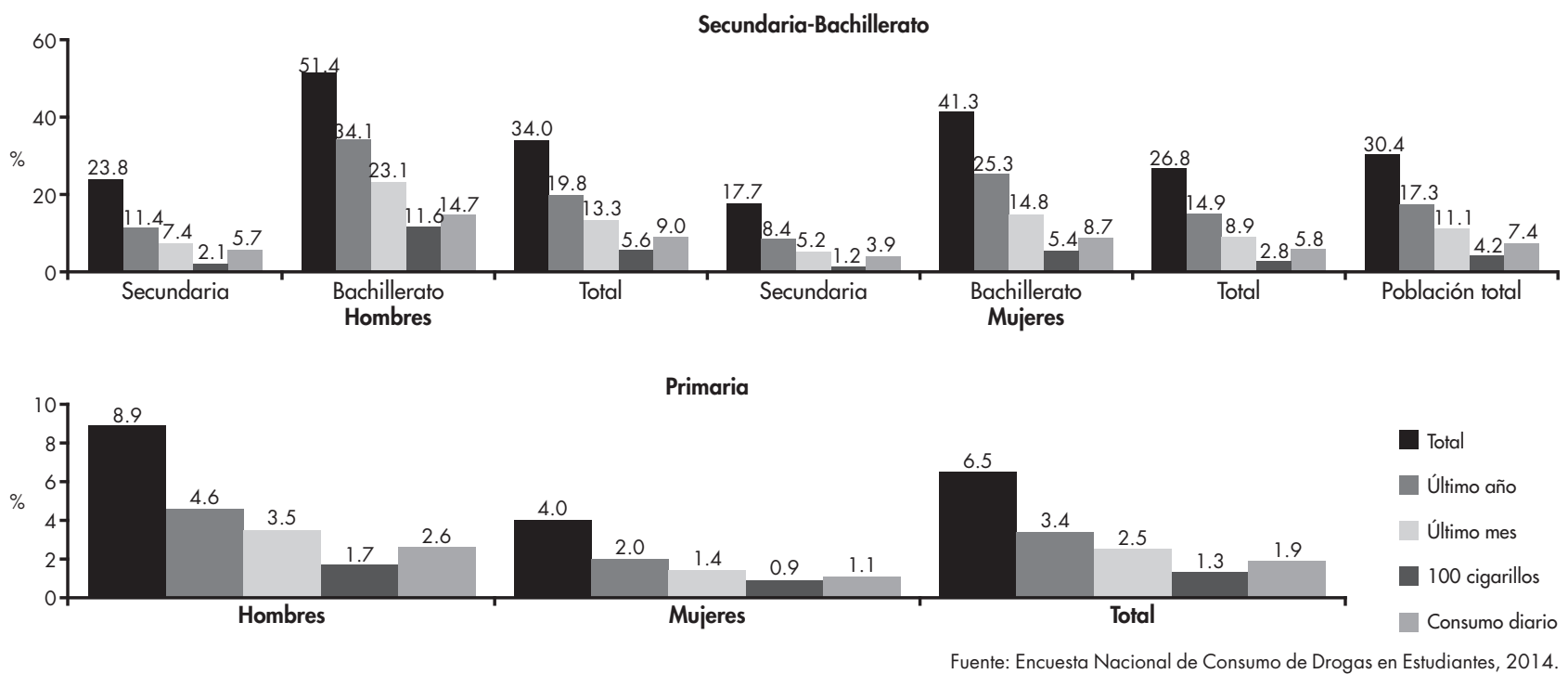

Figura 4. Prevalencia del consumo del tabaco en estudiantes.

tual, $3.5 \%$ son hombres y $1.4 \%$ son mujeres; así, los Estados que reportan mayor porcentaje de fumadores actuales son Querétaro y Quintana Roo (4.9\% cada uno) (cuadro 3).

El $1.9 \%$ indica que fuma diariamente, $2.6 \%$ hombres y $1.1 \%$ mujeres.

Cuando se analiza el consumo por tipo de comunidad, se observa que los estudiantes de primaria de escuelas en comunidades urbanas y rurales tienen un consumo similar en la prevalencia "alguna vez" (6.5\% urbanas, 6.7\% rurales), así como en los indicadores de consumo en el último año (3.3\% urbanas, 3.4\% rurales), en el último mes (2.5\% urbanas, $2.6 \%$ rurales), en el consumo de 100 cigarrillos o más (1.2\% urbanas, $1.6 \%$ rurales) y el consumo diario $(1.9 \%$ en ambos tipos de comunidad).

Acerca de la intención de consumir tabaco, el 11.6\% de estos estudiantes dijeron que tenían la intención de fumar por primera vez y en los estudiantes que ya han fumado por primera vez, $44.5 \%$ menciona tener la intención de volverlo a hacer.

\section{Prevalencias del consumo de drogas}

Los resultados de la Encuesta Nacional de Consumo de Drogas en Estudiantes (ENCODE) muestran que a nivel nacional el $17.2 \%$ de los jóvenes de secundaria y bachillerato han consumido drogas alguna vez en la vida, en los hombres $18.6 \%$ y en las mujeres $15.9 \%$; particularmente, por nivel educativo la prevalencia cambia de $12.5 \%$ en secundaria a $25.1 \%$ en bachillerato. Las prevalencias estatales de consumo más altas las encontramos en Ciudad de México (25\%), Quintana Roo (22\%), Estado de México (21.1\%), Jalisco $(20.1 \%)$ y Chihuahua (19.8\%) (cuadro 1$)$.

Las drogas de preferencia entre los adolescentes son la mariguana (10.6\%), seguida de los inhalables $(5.8 \%)$ y la cocaína (3.3\%). Los Estados con prevalencias mayores a la nacional en el consumo de mariguana "alguna vez" son Distrito Federal (18.2\%), Quintana Roo (14.6\%) y Estado de México (13.8\%). Para el consumo de cocaína son Distrito Federal (5.2\%) y Quintana Roo (4.7\%). En cuanto a inhalables, el Estado de México (8.8\%) y Distrito Federal (8\%) presentan prevalencias mayores a la nacional (cuadro 1).

La edad de inicio en el consumo de drogas fue de 13 años, edad similar entre hombres y mujeres (13 años cada uno respectivamente).

La prevalencia anual de cualquier droga es de $12.2 \%$ (13.2\% en hombres y $11.2 \%$ en mujeres), en secundaria es de $8.9 \%$ y para bachillerato el porcentaje se incrementa a $17.6 \%$. Por sustancia específica, $7.6 \%$ consumió mariguana en el último año (9.2\% en hombres y $6.1 \%$ en mujeres); $1.7 \%$ cocaína (hombres $2.2 \%$ y mujeres $1.2 \%$ ) y $3.6 \%$ inhalables (3.6\% hombres y $3.6 \%$ mujeres).

Al comparar el consumo de drogas entre los estudiantes de escuelas en comunidades urbanas y rurales, se observa que la prevalencia total en las comunidades rurales es de $11.2 \%$, significativamente menor que el $18.3 \%$ de las escuelas en comunidades urbanas. Esta situación es similar para todas las drogas, particularmente en mariguana $(11.6 \%$ en urbanas y $5.2 \%$ en rurales), seguida por los inhalables (6.2\% y $3.9 \%$, respectivamente) (cuadro 4 ).

Considerando los resultados del ASSIST, * el 1.3\% (1.5\% hombres, $1 \%$ mujeres) requiere tratamiento especializado por su consumo de drogas (cuadro 2).

Por otro lado, se muestra que a nivel nacional las prevalencias de consumo tuvieron un crecimiento entre 1991

* El ASSIST permite obtener dos categorías de apoyo o tratamiento: apoyo o intervención breve y apoyo o tratamiento especializado. Esta última categoría presenta un porcentaje bajo a nivel nacional, que es de $1.3 \%$. 
Cuadro 4. Indicadores de consumo de sustancias psicoactivas por tipo de comunidad en estudiantes de secundaria y bachillerato

\begin{tabular}{|c|c|c|c|c|c|c|}
\hline & \multicolumn{2}{|c|}{ Hombre } & \multicolumn{2}{|c|}{ Mujer } & \multicolumn{2}{|c|}{ Total } \\
\hline & Urbana & Rural & Urbana & Rural & Urbana & Rural \\
\hline Alguna vez & $\%$ & $\%$ & $\%$ & $\%$ & $\%$ & $\%$ \\
\hline Cualquier droga & 19.5 & 13.5 & 17.1 & 8.8 & 18.3 & 11.2 \\
\hline Drogas médicas* & 5.2 & 4.3 & 6.9 & 3.9 & 6.1 & 4.1 \\
\hline Drogas ilegales & 17.6 & 11.0 & 13.6 & 6.5 & 15.6 & 8.8 \\
\hline Anfetaminas * & 2.3 & 2.1 & 2.9 & 1.8 & 2.6 & 2.0 \\
\hline Tranquilizantes* & 3.3 & 2.5 & 4.6 & 2.4 & 4.0 & 2.4 \\
\hline Mariguana & 13.9 & 7.1 & 9.3 & 3.2 & 11.6 & 5.2 \\
\hline Cocaína & 4.4 & 2.8 & 2.7 & 1.2 & 3.5 & 2.0 \\
\hline Crack & 2.3 & 1.3 & 1.2 & 0.7 & 1.7 & 1.0 \\
\hline Alucinógenos & 2.7 & 1.4 & 1.5 & 0.8 & 2.1 & 1.1 \\
\hline Inhalables & 6.2 & 4.4 & 6.2 & 3.4 & 6.2 & 3.9 \\
\hline Metanfetaminas & 2.4 & 1.6 & 1.8 & 1.1 & 2.1 & 1.3 \\
\hline Heroína & 1.0 & 1.2 & 0.7 & 0.6 & 0.9 & 0.9 \\
\hline
\end{tabular}
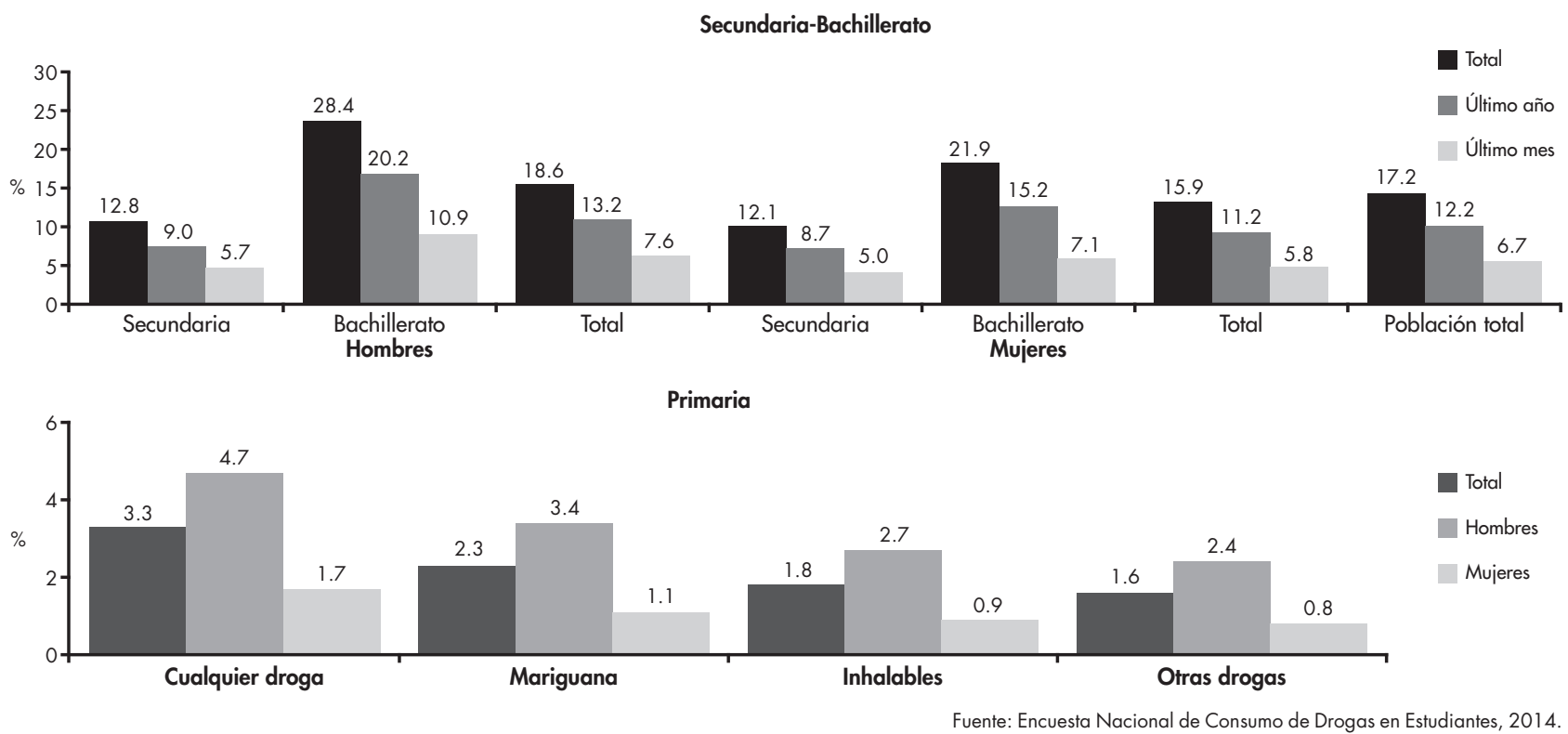

Figura 5. Prevalencia del consumo de drogas en estudiantes.

y 2014; el consumo de drogas alguna vez pasó de $8.2 \%$ a $17.2 \%$; el consumo de mariguana aumentó de $1.5 \%$ a $10.6 \%$, los inhalables de $3.5 \%$ a $5.8 \%$ y la cocaína de $0.7 \%$ a $3.3 \%$ (figura 3).

El consumo "alguna vez en la vida" de drogas en los estudiantes de $5^{\circ}$ y $6^{\circ}$ de primaria es de $3.3 \%$ (hombres $4.7 \%$ y mujeres 1.7\%) (figura 5). Los Estados con prevalencias mayores al nacional son Quintana Roo (5.9\%), Michoacán (5.3\%), San Luis Potosí (5.1\%) y Campeche (5\%) (cuadro 3). En cuanto al consumo en el último año, éste fue de $2.6 \%$ (3.9\% en hombres y $1.3 \%$ en mujeres) y se presenta una prevalencia mayor al nacional en Quintana Roo (4.9\%).

El uso de mariguana entre los estudiantes de primaria tiene una prevalencia de $2.3 \%$ (3.4\% hombres y $1.1 \%$ mujeres) y el de inhalables de $1.8 \%$ (2.7\% hombres y $0.9 \%$ mujeres). Particularmente en el consumo de mariguana los hombres tienen uno tres veces mayor al de las mujeres (figura 5).

Por su parte, la prevalencia total de consumo de cualquier droga en escuelas que se encuentran en poblaciones urbanas y rurales se presenta de manera similar (3.2\% urbana y $3.5 \%$ rural), esto mismo se presenta para la prevalencia de consumo de mariguana $(2.3 \%$ en ambos tipos de comunidad), inhalables (1.8\% urbana, $2 \%$ rural) y otras drogas (1.6\% urbana, $1.7 \%$ rural).

Respecto a la intención de consumir drogas, el 8.3\% tiene la intención de consumirla por primera vez y de los que ya la consumieron, el 10.7\% quiere volver a hacerlo. 
El consumo en $5^{\circ}$ y $6^{\circ}$ de primaria en su mayoría es experimental, ya que siete de cada 10 usuarios de drogas han consumido entre una y cinco veces.

\section{DISCUSIÓN Y CONCLUSIÓN}

El estudio ha permitido identificar la magnitud del crecimiento del consumo de sustancias en el país. Particularmente, los mayores incrementos se deben al consumo de drogas ilegales y médicas, así como el patrón de consumo excesivo que en el primer caso se ha duplicado y en el segundo creció más del $60 \%$.

Esta tendencia es similar a la observada en las encuestas estatales ${ }^{5}$ que se han hecho en el país y a lo visto en la región de las Américas, ${ }^{10}$ donde se muestra un crecimiento importante del consumo, en particular de la mariguana y el alcohol.

La prevalencia de consumo excesivo en los estudiantes, es de $2.4 \%$ en primaria y $14.5 \%$ en secundaria y bachillerato. Los Estados de Querétaro y Tlaxcala en primaria, así como la Ciudad de México, Durango, Jalisco y Michoacán en secundaria y bachillerato presentan prevalencias mayores al promedio nacional. En cuanto al consumo de drogas, la prevalencia "alguna vez" de cualquier droga en primaria es de $3.3 \%$, en tanto que en secundaria y bachillerato es de 17.2\%. Los Estados con mayores prevalencias fueron San Luis Potosí, Michoacán, Campeche y Quintana Roo en el nivel primaria y Ciudad de México, Estado de México, Jalisco, Chihuahua y Quintana Roo en el nivel de secundaria y bachillerato.

Estos datos dirigen la planeación de la prevención y los servicios de tratamiento, tanto federales como estatales, a los Estados en que se debe intervenir con mayor profundidad y poder disminuir los índices de consumo.

Otra información obtenida del estudio con los niños de primaria, es que el $8.3 \%$ indica que tienen la intención de consumir drogas, $12.5 \%$ para alcohol y $11.6 \%$ para tabaco, y en caso de los que ya han consumido sustancias, la intención de volver a hacerlo es de $10.7 \%$ para drogas, $22.6 \%$ para alcohol y $44.5 \%$ para tabaco. Este dato debe ser considerado en las diversas acciones que se realizan en el país, a fin de que se pueda revertir su interés por iniciarse en el consumo de sustancias y, en esa medida, darles mejores opciones de esparcimiento y desarrollo a nuestras nuevas generaciones.

$\mathrm{Al}$ analizar los comportamientos de consumo por sexo, en la mayoría de los indicadores el consumo de hombres y mujeres es similar en todos los Estados. De hecho, el mayor crecimiento de los últimos 23 años es justamente en las mujeres, ante lo cual se debe intervenir de manera importante a fin de disminuir dicha tendencia. En los estudios de la Ciudad de México, se ha encontrado incluso que en algunas Delegaciones el consumo llega a ser mayor en las estudiantes de secundaria y bachillerato. ${ }^{5}$
Lo anterior está en relación con los resultados encontrados por el tipo de comunidad, en las urbanas, el consumo de alcohol y drogas "alguna vez", así como el consumo de tabaco en el último mes es mayor en comparación con las rurales. Esta situación se refleja en los Estados con mayor población rural; los niveles de consumo en general son menores y la brecha entre el consumo de los hombres y las mujeres es mayor, posiblemente aunado al mantenimiento de una mayor convivencia y comunicación familiar en esas partes del país, que protegen al individuo ante el consumo de sustancias. ${ }^{11}$

En el caso del tabaco, las prevalencias se han mantenido en los últimos 23 años. Si bien también se ha disminuido la diferencia entre hombres y mujeres, el nivel general de consumo se ha mantenido. Esto posiblemente porque la mayor parte de las acciones de prevención en el país se han dirigido a prevenir y a cesar el consumo de tabaco y en algunos casos, como la Ciudad de México, se ha logrado disminuir su prevalencia.

En cuanto a las limitaciones de este estudio, como encuesta probabilística transversal, no es posible analizar el desarrollo de los eventos a lo largo del tiempo, ya que la medición se hace en un solo momento temporal. Por ello, las comparaciones se hacen sobre diversas muestras de sujetos y no los mismos, aunque provengan del mismo tipo de población; en este caso, estudiantes de tres distintos niveles educativos. Dado este contexto, la información, si bien es valiosa, se debe analizar en un contexto de descripción.

Los resultados nos muestran la necesidad de emprender de manera más sistemática las acciones de prevención $\mathrm{y}$ tratamiento; aunque se tienen diversas intervenciones preventivas en el país, es menester poder contar con una política de prevención que lleve a mejores resultados, que dé prioridad a la aplicación de estrategias preventivas probadas y que sus resultados puedan ser sujetos a evaluación. De manera particular, se deben incrementar las intervenciones dirigidas a los grupos de menor edad, de preescolar y primaria, y a sus padres, con objeto de tener mejores resultados en la promoción de la salud mental de nuestra población.

\section{Financiamiento}

La Encuesta Nacional de Consumo de Drogas en Estudiantes fue financiada por la Comisión Nacional Contra las Adicciones (CONADIC) y la Secretaría de Salud.

\section{Conflicto de intereses}

Los autores declaran no tener ningún conflicto de intereses.

\section{Agradecimientos}

Queremos agradecer a la Secretaría de Educación Pública (SEP) y a las Secretarías de Educación Estatales que nos ayudaron constantemente para facilitar el acceso a las escuelas en cada uno de los Estados. De manera particular, agradecemos al maestro Antonio Ávila, 
quien coordinó al grupo de la SEP. A los subsecretarios de Planeación, de Educación Media Superior y de Educación Básica, que por medio de sus representantes, Cynthia María Elizabeth Cabrera Cárdenas, Georgina Islas Puente, Paula Villaseñor Torres y Sergio Fernández Vargas Téllez, apoyaron en forma fundamental el proyecto. Por su apoyo en diversas fases del proyecto y su profesionalismo, agradecemos a Natania Oliva, Jessica Merino, Michelle Bretón, Clara Fleiz, Araceli Gutiérrez, Mitzi Razo, Viridiana López, Arlem Solares, Lydia Mendoza y Ma. de Lourdes Gutiérrez López.

De la misma forma, agradecemos al doctor Mario Gómez Espinosa su tiempo y apoyo incondicional para este proyecto.

A Ricardo Nanni, José Oliver Galeana M., Héctor Martínez y a Alfredo González, de la CONADIC, a Rafael Gutiérrez y a Leticia Vega, les agradecemos su disposición y aprendizajes en el proceso del estudio. También agradecemos al grupo de la Dirección General de Epidemiología (doctor Arturo Revuelta, maestro Mario Cortés y doctor Pedro Murguía), por su labor en la evaluación, seguimiento y por sus valiosos comentarios que mejoraron el presente proyecto. A todos quienes participaron en el estudio, mil gracias por su apoyo y compromiso.

\section{REFERENCIAS}

1. Secretaría de Salud, Subsecretaría de Prevención y Promoción de la Salud, Dirección General de Epidemiología. Sistema de Vigilancia Epidemiológica de las Adicciones (SISVEA) Informe 2012; 2013.

2. Ortiz A, Martínez R, Meza D. Grupo Interinstitucional para el desarrollo del Sistema de Reporte de Información en Drogas. Resultados de la aplicación de la cédula: "Informe individual sobre consumo de drogas". Tendencias en el área metropolitana No. 55, noviembre de
2013. Ciudad de México: Instituto Nacional de Psiquiatría Ramón de la Fuente Muñiz; septiembre; 2014.

3. Medina-Mora ME, Rojas E, Juarez F, Berenzon S et al. Encuesta nacional sobre el uso de drogas entre la comunidad escolar 1991. Ciudad de México; SEP, INPRFM; 1991.

4. United Nations Office on Drugs and Crime, World Drug Report 2014 (United Nations publication, Sales No. E.14.XI.7); junio 2014 Disponible en: https://www.unodc.org/documents/wdr2014/World_Drug_Report_2014_web.pdf

5. Villatoro J, Oliva N, Mujica R, Fregoso D et al. Panorama Actual del consumo de sustancias en estudiantes de la Ciudad de México. Ciudad de México: Instituto Nacional de Psiquiatría Ramón de la Fuente Muñiz, Administración Federal de Servicios Educativos en el Distrito Federal, Instituto para la Atención y Prevención de las Adicciones; 2015.

6. Villatoro-Velázquez JA, Oliva Robles N, Fregoso Ito D, Bustos Gamiño $\mathrm{M}$ et al. Encuesta nacional de consumo de drogas en estudiantes 2014. Reporte de drogas. Ciudad de México: Instituto Nacional de Psiquiatría Ramón de la Fuente Muñiz, Comisión Nacional Contra las Adicciones, Secretaría de Salud; 2015.

7. López E, Medina-Mora ME, Villatoro J, Juárez F et al. Factores relacionados al consumo de drogas y al rendimiento académico en adolescentes. Psicología Social México 1996;6:561-567.

8. Mariño MC, Medina-Mora ME, Chaparro J, González-Forteza C. Confiabilidad y estructura factorial del CES-D en adolescentes mexicanos. Rev Mex Psicol 1993;10(2):141-145.

9. Medina-Mora ME, Castro S, Gómez-Mont F, Campillo C. Validity and reliability of a high school drug use questionnaire among Mexican students. Bull Narc 1981;33(4):67-76.

10. Organización de los Estados Americanos OEA, Comisión Interamericana para el Control del Abuso de Drogas. Informe sobre uso de drogas en las Américas. Washington DC: 2015.

11. Martínez JM, Robles L. Variables de protección ante el consumo de alcohol y tabaco en adolescentes. Psicothema 2001;13(2):222-228. 